\title{
Degrees of Freedom of the MIMO $X$ Channel
}

\author{
Syed A. Jafar \\ Electrical Engineering and Computer Science \\ University of California Irvine, \\ Irvine, California, 92697, USA \\ Email: syed@uci.edu
}

\author{
Shlomo Shamai (Shitz) \\ Department of Electrical Engineering \\ Technion-Israel Institute of Technology \\ Technion City, Haifa 32000, Israel \\ Email: sshlomo@ee.technion.ac.il
}

\begin{abstract}
We provide achievability as well as converse results for the degrees of freedom region of a MIMO $X$ channel, i.e., a system with two transmitters, two receivers, each equipped with multiple antennas, where independent messages need to be conveyed over fixed channels from each transmitter to each receiver. The inner and outerbounds on the degrees of freedom region are tight whenever integer degrees of freedom are optimal for each message. If all nodes have equal number of antennas $M>1$ and channel matrices are non-degenerate then the degrees of freedom $\eta_{X}^{\star}=\frac{4}{3} M$. If the channels vary with time/frequency then the $X$ channel with single antennas $(M=1)$ at all nodes has $4 / 3$ degrees of freedom. Thus, the MIMO $X$ channel has non-integer degrees of freedom when $M$ is not a multiple of 3 . Simple zero forcing without dirty paper encoding or successive decoding, suffices to achieve the $\frac{4}{3} M$ degrees of freedom in all cases. The key idea for the achievability of the degrees of freedom is interference alignment - i.e., signal spaces are aligned at receivers where they constitute interference while they are separable at receivers where they are desired. With equal number of antennas at all nodes, we also explore the increase in degrees of freedom when some of the messages are made available to a transmitter or receiver in the manner of cognitive radio.
\end{abstract}

\section{INTRODUCTION}

Previous work by several researchers has determined the degrees of freedom for various multiuser MIMO systems. The single user point to point MIMO channel with $M_{1}$ transmit and $N_{1}$ receive antennas is known to have $\min \left(M_{1}, N_{1}\right)$ degrees of freedom [1]. For the two user MIMO multiple access channel (MAC) with $N_{1}$ receive antennas and $M_{1}, M_{2}$ transmit antennas at the two transmitters, the maximum multiplexing gain is $\max \left(M_{1}+M_{2}, N_{1}\right)$ [2]. Thus, the multiplexing gain is the same as the point to point MIMO channel with full cooperation among all transmit antennas. The two user broadcast channel (BC) with $M_{1}$ transmit antennas and $N_{1}, N_{2}$ receive antennas has a maximum multiplexing gain of $\max \left(M_{1}, N_{1}+N_{2}\right)$ which is also the same as the point to point MIMO channel obtained with full cooperation between the two receivers [3][5]. The multiplexing gain for two user MIMO interference channels is found in [6]. It is shown that for a $\left(M_{1}, N_{1}, M_{2}, N_{2}\right)$ MIMO interference channel (i.e. a
MIMO interference channel with $M_{1}, M_{2}$ antennas at the two transmitters and $N_{1}, N_{2}$ antennas at their respective receivers), the maximum multiplexing gain is equal to $\min \left(M_{1}+M_{2}, N_{1}+N_{2}, \max \left(M_{1}, N_{2}\right), \max \left(M_{2}, N_{1}\right)\right)$.

In this work we provide achievability as well as converse arguments for the degrees of freedom region of a MIMO $X$ channel, i.e., a system with two transmitters, two receivers, each equipped with multiple antennas, where independent messages need to be conveyed from each transmitter to each receiver. We also consider the benefits of cognitive message sharing at the transmitters and/or receivers for the MIMO $X$ and interference channels.

\section{A. The MIMO X channel}

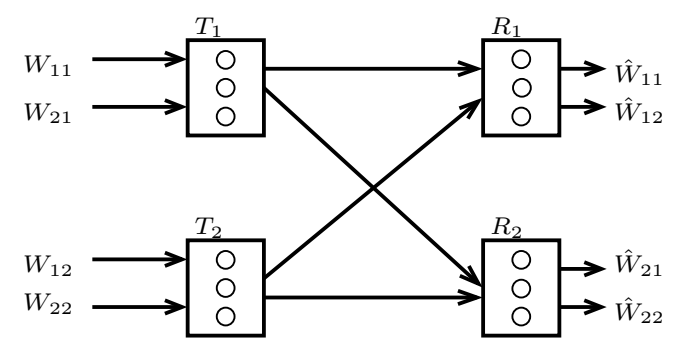

Fig. 1. MIMO $X$ Channel

The MIMO $X$ channel is shown in Figure 1 and is described by the input output equations:

$$
\begin{aligned}
\mathbf{Y}^{[1]} & =\mathbf{H}^{[11]} \mathbf{X}^{[1]}+\mathbf{H}^{[12]} \mathbf{X}^{[2]}+\mathbf{N}^{[1]} \\
\mathbf{Y}^{[2]} & =\mathbf{H}^{[21]} \mathbf{X}^{[1]}+\mathbf{H}^{[22]} \mathbf{X}^{[2]}+\mathbf{N}^{[2]}
\end{aligned}
$$

where $\mathbf{Y}^{[1]}$ is the $N_{1} \times 1$ output vector at receiver 1 , $\mathbf{Y}^{[2]}$ is the $N_{2} \times 1$ output vector at receiver $2, \mathbf{N}^{[1]}$ is the $N_{1} \times 1$ additive white Gaussian noise (AWGN) vector at receiver $1, \mathbf{N}^{[2]}$ is the $N_{2} \times 1$ AWGN vector at receiver $2, \mathbf{X}^{[1]}$ is the $M_{1} \times 1$ input vector at transmitter $1, \mathbf{X}^{[2]}$ is the $M_{2} \times 1$ input vector at transmitter $2, \mathbf{H}^{[11]}$ is the $N_{1} \times M_{1}$ channel matrix between transmitter 1 and receiver $1, \mathbf{H}^{[22]}$ is the $N_{2} \times M_{2}$ channel matrix between transmitter 2 and receiver $2, \mathbf{H}^{[12]}$ is the $N_{1} \times M_{2}$ channel matrix between transmitter 2 and receiver 1 , 
and $\mathbf{H}^{[21]}$ is the $N_{2} \times M_{1}$ channel matrix between transmitter 1 and receiver 2 . As shown in Figure 1 there are four independent messages in the MIMO $X$ channel: $W_{11}, W_{12}, W_{21}, W_{22}$ where $W_{i j}$ represents a message from transmitter $j$ to receiver $i$.

We assume the channel matrices are generated from a continuous probability distribution so that, almost surely, any matrix composed of channel coefficients will have rank equal to the minimum of the number of its rows and columns. Perfect knowledge of all channel coefficients is available to all transmitters and receivers. With the exception of Theorem 5, we assume throughout that the values of the channel coefficients are fixed throughout the duration of communication.

The sum power of all transmitters is assumed to be equal to $\rho$. We indicate the size of the message set by $\left|W_{i j}(\rho)\right|$. For codewords spanning $n$ channel uses, the rates $R_{i j}(\rho)=\frac{\log \left|W_{i j}(\rho)\right|}{n}$ are achievable if the probability of error for all messages can be simultaneously made arbitrarily small by choosing an appropriately large $n$. The capacity region $\mathcal{C}^{X}(\rho)$ of the $X$ channel is the set of all achievable rate tuples $\mathbf{R}(\rho)=\left(R_{11}(\rho), R_{12}(\rho), R_{21}(\rho), R_{22}(\rho)\right)$.

We define the degrees of freedom region for the MIMO $X$ channel as:

$\mathcal{D}^{X}=\left\{\left(d_{11}, d_{12}, d_{21}, d_{22}\right) \in \mathbb{R}_{+}^{4}: d_{i j}=\lim _{\rho \rightarrow \infty} \frac{R_{i j}(\rho)}{\log (\rho)}\right\}$

The total degrees of freedom $\eta_{X}^{\star}$ is defined as:

$$
\eta_{X}^{\star} \triangleq \max _{\mathcal{D}^{X}}\left(d_{11}+d_{12}+d_{21}+d_{22}\right)
$$

The MIMO $X$ channel is especially interesting because it is generalizes the interference channel to allow an independent message from each transmitter to each receiver. An interesting coding scheme is recently proposed by Maddah-Ali, Motahari and Khandani in [7] for the two user MIMO $X$ channel with three antennas at all nodes. Just as the MIMO $X$ channel combines elements of the MIMO broadcast channel, the MIMO multiple access channel and the MIMO interference channel into one channel model, the MMK scheme naturally combines dirty paper coding, successive decoding and zero forcing elements into an elegant coding scheme tailored for the MIMO $X$ channel. The results of [6] establish that with 3 antennas at all nodes, the maximum multiplexing gain for each of the MIMO IC, MAC and $\mathrm{BC}$ channels contained within the $X$ channel is 3 . However, for the MIMO $X$ channel with 3 antennas at all nodes, the MMK scheme is able to achieve 4 degrees of freedom. The MMK scheme also extends easily to achieve $\lfloor 4 M / 3\rfloor$ degrees of freedom on the MIMO $X$ channel with $M$ antennas at each node. Thus, the results of [7] show that the degrees of freedom on the MIMO $X$ channel strictly surpass what is achievable on the interference, multiple access and broadcast components individually.

Several interesting questions arise for the MIMO $X$ channel. First, we need an outerbound to determine what is the maximum multiplexing gain for the MIMO $X$ channel, and in particular, if the MMK scheme is optimal. Second, we note that neither dirty paper coding nor successive decoding have been found to be necessary to achieve the full degrees of freedom on any multiuser MIMO channel with perfect channel knowledge. Zero forcing suffices to achieve all degrees of freedom on the MIMO MAC, BC, and interference channels. So the natural question is whether zero forcing also suffices to achieve all the degrees of freedom for the MIMO $X$ channel. Third, we note that there are no known results for the optimality of non-integer degrees of freedom for any non-degenerate wireless network with perfect channel knowledge. The results of [7] have lead to the conjecture that $\lfloor 4 / 3 M\rfloor$ is the optimal number of degrees of freedom for the MIMO $X$ channel with $M$ antennas at each node, which reinforces the intuition that degrees of freedom must take integer values. It is therefore of fundamental interest to determine if this intuition is correct or if indeed noninteger degrees of freedom can be optimal for the $X$ channel. Finally, while the interference channel does not seem to benefit from cooperation through noisy channels between transmitters and receivers, it is not known if shared messages (in the manner of cognitive radio [8]) can improve the degrees of freedom on the MIMO $X$ and interference channels. These are the open questions that we answer in this work.

Notation: $\operatorname{co}(\mathrm{A})$ is the convex hull of the set $\mathrm{A} . \mathbb{R}_{+}^{n}$ and $\mathbb{Z}_{+}^{n}$ represent the set of n-tuples of non-negative real numbers and integers respectively.

\section{MAIN ReSUlts}

We summarize the main results of this work in Theorems 1 to 5. Due to space constraints, we refer the reader to the full paper [9] for the detailed proofs.

Theorem 1: $\mathcal{D}^{X} \subset \mathcal{D}_{\text {out }}^{X}$ where the outerbound on the degrees of freedom region is defined as follows.

$$
\begin{aligned}
\mathcal{D}_{\text {out }}^{X} \triangleq & \left\{\left(d_{11}, d_{12}, d_{21}, d_{22}\right) \in \mathbb{R}_{+}^{4}:\right. \\
& d_{11}+d_{12}+d_{21} \leq \max \left(N_{1}, M_{1}\right) \\
& d_{11}+d_{12}+d_{22} \leq \max \left(N_{1}, M_{2}\right) \\
& d_{11}+d_{21}+d_{22} \leq \max \left(N_{2}, M_{1}\right) \\
& d_{12}+d_{21}+d_{22} \leq \max \left(N_{2}, M_{2}\right) \\
& d_{11}+d_{12} \leq N_{1} \\
& d_{21}+d_{22} \leq N_{2}
\end{aligned}
$$




$$
\begin{aligned}
& d_{11}+d_{21} \leq M_{1} \\
& \left.d_{12}+d_{22} \leq M_{2}\right\}
\end{aligned}
$$

Note that the outerbound allows all real non-negative values for $d_{i j}$ that satisfy the 8 inequalities. The boundary values of $d_{i j}$, e.g., those that maximize $\eta_{X}$ may not be integers. This is the main distinction between the outerbound and the innerbound presented in Theorem 3 .

While the set $\mathcal{D}_{\text {out }}^{X}$ provides an outerbound for all achievable $d_{i j}$ on the MIMO $X$ channel, maximizing any weighted sum of $d_{i j}$ over $\mathcal{D}_{\text {out }}^{X}$ is a linear programming problem. The following theorem presents an outerbound $\eta_{\text {out }}$ for the total degrees of freedom $\eta_{X}^{\star}$ in closed form by explicitly solving the linear programming problem.

Theorem 2:

$$
\begin{gathered}
\eta_{\text {out }} \triangleq \max _{\mathcal{D}_{\text {out }}^{X}}\left(d_{11}+d_{12}+d_{21}+d_{22}\right) \\
=\min \left\{M_{1}+M_{2}, N_{1}+N_{2}, \frac{\max \left(M_{1}, N_{1}\right)+\max \left(M_{1}, N_{2}\right)+M_{2}}{2},\right. \\
\frac{\max \left(M_{2}, N_{1}\right)+\max \left(M_{2}, N_{2}\right)+M_{1}}{2}, \\
\frac{\max \left(M_{1}, N_{1}\right)+\max \left(M_{2}, N_{1}\right)+N_{2}}{2}, \\
\frac{\max \left(M_{1}, N_{2}\right)+\max \left(M_{2}, N_{2}\right)+N_{1}}{2}, \\
\left.\frac{\max \left(M_{1}, N_{1}\right)+\max \left(M_{1}, N_{2}\right)+\max \left(M_{2}, N_{1}\right)+\max \left(M_{2}, N_{2}\right)}{3}\right\}
\end{gathered}
$$

Note that all 7 terms in the min expression of Theorem 2 are necessary in general. The following examples with different values of $\left(M_{1}, M_{2}, N_{1}, N_{2}\right)$ illustrate this point, as in each case only one of the 7 bounds is tight.

$$
\begin{array}{cl}
(1,1,2,2) & \Rightarrow \eta_{\text {out }}=2 \\
(4,8,6,10) & \Rightarrow \eta_{\text {out }}=11 \\
(3,3,3,3) & \Rightarrow \eta_{\text {out }}=4 .
\end{array}
$$

For completeness, we now state the innerbound from [10], [11]. An alternate constructive proof of this innerbound is also presented in [9].

Theorem 3: $\mathcal{D}^{X} \supset \mathcal{D}_{\text {in }}^{X} \triangleq \operatorname{co}\left(\mathcal{D}_{\text {out }}^{X} \cap \mathbb{Z}_{+}^{4}\right)$.

Several interesting observations can be made regarding the schemes used in [9], [11], [12] to establish the achievable degrees of freedom for the MIMO $X$ channel. First, these schemes do not require dirty paper coding or successive decoding. Instead, as with the MIMO MAC, $\mathrm{BC}$ and interference channels, the optimal achievability schemes are based on simple zero forcing. The distinguishing feature of the MIMO $X$ channel is the concept of interference alignment. Interference alignment refers to the careful choice of beamforming directions in such a manner that the desired signals are separable at their respective receivers while the interference signals are aligned, i.e., the interference vectors cast overlapping shadows. Overlapping of interference subspaces is pointed out as a useful idea for the MIMO $X$ channel by Maddah-Ali, Motahari and Khandani in [10]. The first explicit interference alignment scheme is developed in
[12] for the 2 user $X$ channel. Interference alignment is subsequently shown to achieve all the points within the innerbound of Theorem 3 in [11], [9]. Interference alignment is also found to be useful for the compound broadcast channel in [13].

It has been conjectured that the integer innerbound of Theorem 3 is the optimal degree of freedom region for the MIMO $X$ channel. Interestingly, we show in this paper that the integer degrees of freedom innerbound is not optimal. In fact it is the outerbound that is tight in most cases as the optimal degrees of freedom take noninteger values. For example consider the MIMO $X$ channel with $M$ antennas at each node (where $M$ is not a multiple of 3). The maximum number of degrees of freedom according to the integer innerbound equals $\lfloor 4 M / 3\rfloor$ while according to the outerbound it is $4 M / 3$. For example, with $M=2$ the integer innerbound leads to only 2 degrees of freedom while the outerbound suggests $2.66 \cdots$ degrees of freedom. As we show in Theorem 4 for all cases with $M>1$ antennas at all nodes, it is the outerbound that is tight.

Theorem 4: For the MIMO $X$ channel with equal number of antennas $M_{1}=M_{2}=N_{1}=N_{2}=M>1$ antennas at all nodes the degrees of freedom

$$
\eta^{\star}=\frac{4}{3} M
$$

The key to the proof is to consider a 3 symbol extension of the channel so that we have effectively a $3 M \times 3 M$ channel, over which we will achieve $4 M$ degrees of freedom. Note that we still assume the channel matrices are fixed, so that the 3 symbol extension does not provide us a new channel matrix over each slot.

For $M=1$ we are also able to achieve the full $4 / 3$ degrees of freedom if the channel coefficients are time/frequency selective.

Theorem 5: For the MIMO $X$ channel with single antenna $\left(M_{1}=M_{2}=N_{1}=N_{2}=1\right)$ at all nodes the spatial degrees of freedom $\eta^{\star}=\frac{4}{3}$ per orthogonal time/frequency dimension if the channel coefficients are time/frequency varying and drawn independent and identically distributed from a continuous distribution.

\section{COOPERATION THROUGH INTERFERENCE ALIGNMENT AND SHARED MESSAGES}

Message sharing for the interference channel with single antenna nodes has been studied recently by Devroye and Sharif in [14] in the context of cognitive radio. Exploring this idea further, we characterize the degrees of freedom for the MIMO cognitive radio channel, i.e. the MIMO interference channel when some messages are made available in the manner of cognitive radio at either the transmitters or the receivers. For simplicity we 


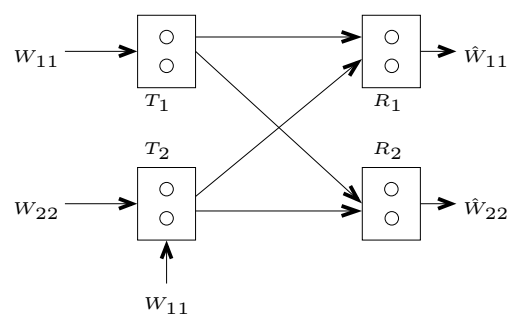

(a)

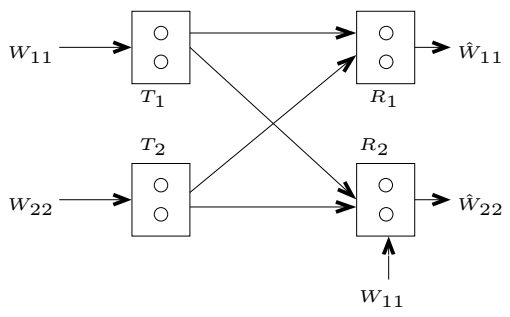

(b)

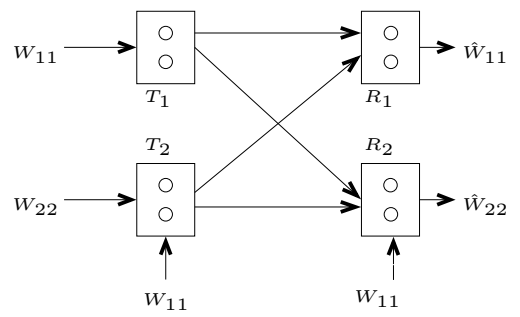

(c)

Fig. 2. Cognitive MIMO Interference Channels with $\eta^{\star}=M$.

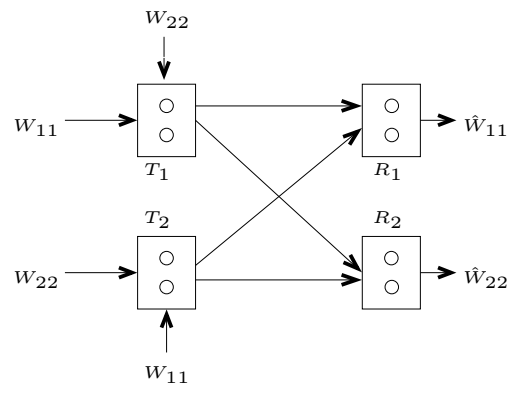

(a)

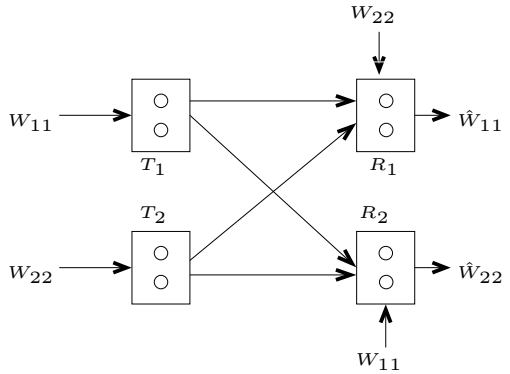

(b)

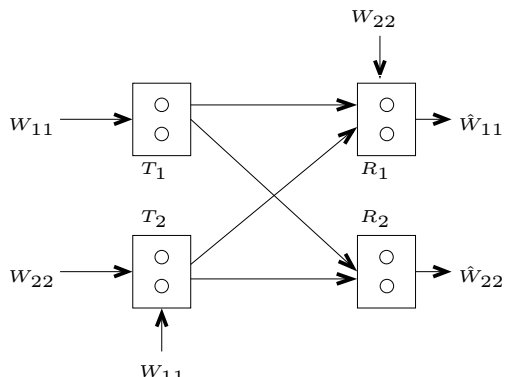

(c)

Fig. 3. Cognitive MIMO Interference Channels with $\eta^{\star}=2 M$.

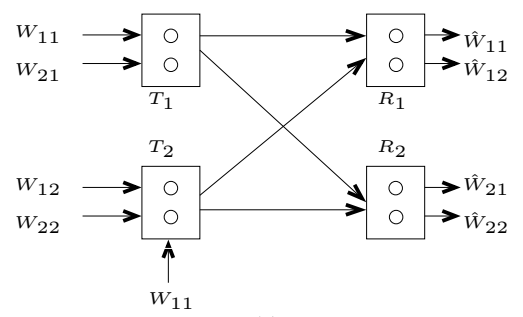

(a)

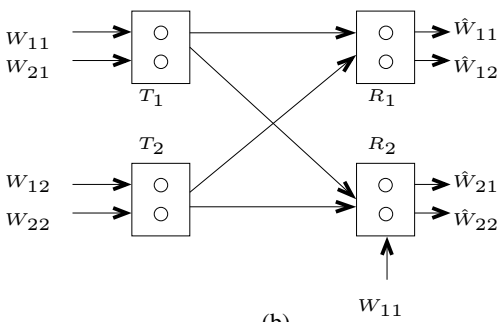

(b)

Fig. 4. MIMO $X$ Channels with (a) Cognitive Transmitter and (b) Cognitive Receiver. In both cases, $\eta^{\star}=\frac{3}{2} M$ for $M>1$.

focus primarily on the cases with $M_{1}=M_{2}=N_{1}=$ $N_{2}=M$.

\section{A. Cognitive MIMO interference channel}

We refer to the interference channel with one message shared between the two transmitters as the interference channel with a cognitive transmitter. This channel is often referred to as the cognitive radio channel and is shown in Figure 2. Following cognitive radio terminology, transmitter 1 is the primary transmitter whose message for primary receiver (receiver 1) is known noncausally to transmitter 2 , the secondary transmitter. Similarly, an interference channel with a cognitive receiver could be defined as the scenario when the primary user's message is known to the secondary user's receiver. Then there is also the possibility that both the secondary transmitter and secondary receiver have knowledge of the primary user's message. Theorems 6 and 7 establish the total degrees of freedom on the MIMO interference channel with cognitive transmitters and/or receivers.

Theorem 6: On the MIMO interference channel with equal number $(M)$ of antennas at all nodes, the total number of degrees of freedom is equal to $M$ for each of the following cognition scenarios (shown in Fig. 2).

1) $W_{11}$ is made available non-causally to transmitter 2 (Fig. 2(a)).

2) $W_{11}$ is made available to receiver 2 (Fig. 2(b)).

3) $W_{11}$ is made available non-causally to transmitter 2 and also to receiver 2 (Fig. 2(c)).

Thus, there is no benefit (in terms of total degrees of freedom) from sharing one user's message on the MIMO interference channel with equal number of antennas at all nodes even if this message is shared with both the transmitter and receiver of the other user. However, if 
the nodes have different number of antennas then the MIMO interference channel may indeed benefit from cognitive message sharing. A simple example is the $(1, n, n, 1)$ case, i.e. transmitter 1 and receiver 2 have one antenna each while receiver 1 and transmitter 2 have $n>$ 1 antennas. Without message sharing this interference channel has at most 1 degree of freedom. However, if transmitter 1's message $W_{11}$ is made available noncausally to transmitter 2 , then clearly $n$ degrees of freedom can be achieved quite simply by transmitter 2 sending $W_{11}$ to receiver 1 on the $n \times n$ channel between them. Message sharing can also be useful for the interference channel even with single antennas at all nodes when channel matrices take certain specialized structured forms as in [15].

Theorem 7: On the MIMO interference channel with equal number $(M)$ of antennas at all nodes, the total number of degrees of freedom is equal to $2 M$ for each of the following cognition scenarios (shown in Fig. 3).

1) $W_{11}$ is made available non-causally to transmitter 2 and $W_{22}$ is made available non-causally to transmitter 1 (Fig. 3 (a)).

2) $W_{11}$ is made available to receiver 2 and $W_{22}$ is made available to receiver 1 (Fig. 3 (b)).

3) $W_{11}$ is made available non-causally to transmitter 2 and $W_{22}$ is made available to receiver 1 (Fig. 3 (c)).

Next we explore the degrees of freedom of the MIMO $X$ channel with cognitive transmitters or receivers.

\section{B. Cognitive MIMO X channel}

Theorem 8: On the MIMO $X$ channel with $M>1$ antennas at all nodes, the total number of degrees of freedom is equal to $\frac{3}{2} M$ for each of the following cognition scenarios (shown in Fig. 4).

1) $W_{11}$ is made available non-causally to transmitter 2 (Fig. 4 (a)).

2) $W_{11}$ is made available non-causally to receiver 2 (Fig. 4 (b)).

Note that Fig. 4(a) is equivalent to the cognitive $X$ channel in [14]. It is interesting to note that the degrees of freedom for the MIMO $X$ channel increase according to $\frac{4}{3} M$ for no shared messages $\rightarrow \frac{3}{2} M$ for one shared message $\rightarrow \frac{2}{1} M$ for two shared messages (provided the two shared messages are not intended for the same receiver). The symmetry of the results for degrees of freedom with cognitive transmitters and cognitive receivers is also interesting as it points to a reciprocity relationship between the transmitter and receiver side cognitive cooperation.

\section{CONCLUSION}

We characterize the degrees of freedom region for the MIMO $X$ channel, a system with two multiple antenna transmitters and two multiple antenna receivers where independent messages are communicated from each transmitter to each receiver. The $X$ channel is especially interesting because the interference channel, the multiple access channel and the broadcast channels are special cases of this channel. Studying the $X$ channel reveals the importance of interference alignment and multi-letter extensions of the channel, establishes that degrees of freedom can take fractional values, and provides interesting insights into the benefits of cognitive message sharing.

\section{REFERENCES}

[1] G. J. Foschini and M. J. Gans, "On limits of wireless communications in a fading environment when using multiple antennas," Wireless Personal Commun. : Kluwer Academic Press, no. 6, pp. 311-335, 1998.

[2] D. Tse, P. Viswanath, and L. Zheng, "Diversity-multiplexing tradeoff in multiple-access channels," IEEE Trans. Inform. Theory, vol. 50, pp. 1859-1874, Sept. 2004.

[3] W. Yu and J. Cioffi, "Sum capacity of Gaussian vector broadcast channels," IEEE Trans. on Information Theory, vol. 50, pp. 18751892, Sept. 2004.

[4] P. Viswanath and D. Tse, "Sum capacity of the vector Gaussian broadcast channel and uplink-downlink duality," IEEE Trans. Inform. Theory, pp. 1912-1921, Aug 2003.

[5] S. Vishwanath, N. Jindal, and A. Goldsmith, "Duality, achievable rates, and sum-rate capacity of MIMO broadcast channels," IEEE Trans. Inform. Theory, pp. 2895-2909, Oct. 2003.

[6] S. Jafar and M. Fakhereddin, "Degrees of freedom for the MIMO interference channel," in Proc. of ISIT, 2006.

[7] M. Maddah-Ali, A. Motahari, and A. Khandani, "Signaling over MIMO multi-base systems - combination of multi-access and broadcast schemes," in Proc. of ISIT, pp. 2104-2108, 2006.

[8] N. Devroye, P. Mitran, and V. Tarokh, "Achievable rates in cognitive radio channels," IEEE Trans. Inform. Theory., vol. 52, pp. 1813-1827, May 2006.

[9] S. Jafar and S. Shamai, "Degrees of freedom region for the MIMO X channel," in arXiv:cs.IT/0607099v3, May 2007.

[10] M. Maddah-Ali, A. Motahari, and A. Khandani, "Communication over X channel: Signalling and multiplexing gain," in Tech. Report. UW-ECE-2006-12, University of Waterloo, July 2006.

[11] M. Maddah-Ali, A. Motahari, and A. Khandani, "Communication over X channel: Signaling and performance analysis," in Tech. Report. UW-ECE-2006-27, University of Waterloo, December 2006.

[12] S. Jafar, "Degrees of freedom on the MIMO X channel- optimality of the MMK scheme," Tech. Report, Sep. 2006, arXiv:cs.IT/0607099v2.

[13] H. Weingarten, S. Shamai, and G. Kramer, "On the compound MIMO broadcast channel," in Proceedings of Annual Information Theory and Applications Workshop UCSD, Jan 2007.

[14] N. Devroye and M. Sharif, "The multiplexing gain of MIMO $\mathrm{X}$-channels with partial transmit side information," in IEEE Int. Symp. on Info. Theory (ISIT), 2007. Preprint available at the authors' website.

[15] A. Lapidoth, S. Shamai, and M. Wigger, "A linear interference network with local side-information," in IEEE Int. Symp. on Info. Theory (ISIT), 2007. 